\title{
Does sepsis cause increased long-term mortality? a systematic review
}

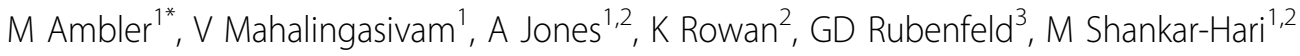 \\ From ESICM LIVES 2015 \\ Berlin, Germany. 3-7 October 2015
}

\section{Introduction}

Acute mortality for adult critically ill patients with sepsis is improving. There is ample evidence that survivors of sepsis have an ongoing risk of death that extends beyond the acute illness [1]. However, whether sepsis is an independent and potentially causal factor in these outcomes is unclear. Since sepsis likely increases acute mortality compared with other conditions, studies to address this question would have to separate acute from post-acute mortality, adjust for confounding variables, and identify appropriate control populations. We performed a systematic review of studies reporting longerterm mortality following sepsis in adult ICU patients to specifically identify those that contained sufficient analyses to address issues of causality.

\section{Objectives}

Our objective was to evaluate whether studies reporting longer-term outcomes have the design features and/or statistical analyses to support causal inferences about the association between sepsis and longer-term mortality.

\section{Methods}

A systematic search for non-randomized and randomized clinical studies in the Medline (1946-2013) and Embase (1974-2013) databases was performed (Ovid platform). Search terms included the following 'mp' terms, MESH headings and combinations thereofsepsis, septic shock, septi?aemia, outcome, quality of life, cohort studies, and randomi?ed controlled trials. Results presented as median [IQR] of proportions. Acute mortality refers to ICU/hospital/up to 30-day

Table 1

\begin{tabular}{|c|c|c|c|c|c|c|c|}
\hline Author [time] & Sepsis subtype & $\begin{array}{l}\text { Acute } \\
\text { Mortality } \\
\text { Sepsis }\end{array}$ & $\begin{array}{l}\text { Post-Acute } \\
\text { Mortality } \\
\text { Sepsis }\end{array}$ & Description of Controls & $\begin{array}{l}\text { Acute } \\
\text { Mortality } \\
\text { Controls }\end{array}$ & $\begin{array}{l}\text { Post-Acute } \\
\text { Mortality } \\
\text { Controls }\end{array}$ & $\begin{array}{l}\text { Analysis } \\
\text { feature for } \\
\text { causality }\end{array}$ \\
\hline $\begin{array}{l}\text { Ghelani } \mathrm{D} \\
\text { et al [5 years] }\end{array}$ & $\begin{array}{l}\text { (a) ICU Admission } \\
\text { (b) Nosocomial }\end{array}$ & $\begin{array}{l}\text { (a) } 41.9 \% \\
\text { (b) } 64.5 \%\end{array}$ & $\begin{array}{l}\text { (a) } 26.5 \% \\
\text { (b) } 15.1 \%\end{array}$ & $\begin{array}{l}\text { (a) Non sepsis ICU } \\
\text { (b) Infected Hospital } \\
\text { (c) Non infected Hospital }\end{array}$ & $\begin{array}{l}\text { (a) } 25.5 \% \\
\text { (b) } 9.5 \% \\
\text { (c) } 2.5 \%\end{array}$ & $\begin{array}{l}\text { (a) } 31.5 \% \\
\text { (b) } 28.6 \% \\
\text { (c) } 18.8 \%\end{array}$ & $\begin{array}{l}\text { Multivariable } \\
\text { adjustment }\end{array}$ \\
\hline $\begin{array}{l}\text { Korosec- } \\
\text { Jagodic } \mathrm{H} \\
\text { et al [2 years] }\end{array}$ & Sepsis & $58.0 \%$ & $11.0 \%$ & Trauma & $38.0 \%$ & $5.0 \%$ & - \\
\hline $\begin{array}{l}\text { Quartin AA } \\
\text { et al [1 year] }\end{array}$ & $\begin{array}{l}\text { (a) Uncomplicated Sepsis } \\
\text { (b) Severe sepsis (c) Septic } \\
\text { shock }\end{array}$ & $\begin{array}{l}\text { (a) } 23.0 \% \\
\text { (b) } 47.0 \% \\
\text { (c) } 57.0 \%\end{array}$ & $\begin{array}{l}\text { (a) } 23.0 \% \\
\text { (b) } 24.0 \% \\
\text { (c) } 23.0 \%\end{array}$ & $\begin{array}{l}3 \text { risk adjusted hospital } \\
\text { controls for each sepsis } \\
\text { subtype }\end{array}$ & $\begin{array}{l}\text { (a) } 8.0 \% \\
\text { (b) } 10.0 \% \\
\text { (c) } 9.0 \%\end{array}$ & $\begin{array}{l}\text { (a) } 16.0 \% \\
\text { (b) } 18.0 \% \\
\text { (c) } 17.0 \%\end{array}$ & $\begin{array}{l}\text { Multivariable } \\
\text { adjustment }\end{array}$ \\
\hline $\begin{array}{l}\text { Regazzoni CJ } \\
\text { et al [1 year] }\end{array}$ & Severe sepsis & $21.8 \%$ & $37.2 \%$ & Non-septic patients & $14.7 \%$ & $20.3 \%$ & $\begin{array}{l}\text { Multivariable } \\
\text { adjustment }\end{array}$ \\
\hline
\end{tabular}


and [1-acute mortality] was defined as acute illness survival. Post-acute mortality is the proportion of deaths in acute illness survivors over the long term follow up period.

\section{Results}

The search identified 4034 articles, excluding duplicates, and 23 studies reported one-year or longer-term, postacute mortality. In these studies, $65.1 \%$ [53.1\%-72.1\%] sepsis patients survived the acute illness. The one-year, post-acute mortality in sepsis survivors was $24.4 \%$ [18.4\% - 42.8\%]. Only four studies reported a non-sepsis control arm and their design/analysis features precluded causal inferences. [2-5]

\section{Conclusions}

Based on our systematic review, there is limited evidence to support the hypothesis that sepsis causes an increase in post-acute mortality. Future studies reporting acute and post-acute mortality, adjusted for confounding, should incorporate multiple control populations to help us answer this question.

\section{Grant Acknowledgment}

NIHR UK, Biomedical Research Centre, Kings Health Partners.

\section{Authors' details}

'Guy's and St Thomas' NHS Foundation Trust, Critical Care Medicine, London, United Kingdom. ${ }^{2}$ Intensive Care National Audit and Research Centre, London, United Kingdom. ${ }^{3}$ Sunnybrook Health Sciences Centre,

Interdepartmental Division of Critical Care Medicine, Toronto, Canada.

Published: 1 October 2015

\section{References}

1. Winters BD, et al: Crit Care Med 2010, 38:1276-83.

2. Ghelani D, et al: J Eval Clin Pract 2009, 15:424-35.

3. Korosec Jagodic $H$, et al: Crit Care 2008, 10:R134.

4. Quartin AA, et al: JAMA 1997, 277:1058-63.

5. Regazzoni CJ, et al: J of Gerontol 2008, 63:210-12.

doi:10.1186/2197-425X-3-S1-A759

Cite this article as: Ambler et al.: Does sepsis cause increased long-term mortality? a systematic review. Intensive Care Medicine Experimental 2015 3(Suppl 1):A759.

\section{Submit your manuscript to a SpringerOpen ${ }^{\mathcal{O}}$ journal and benefit from:}

- Convenient online submission

- Rigorous peer review

- Immediate publication on acceptance

- Open access: articles freely available online

- High visibility within the field

- Retaining the copyright to your article

Submit your next manuscript at $>$ springeropen.com 